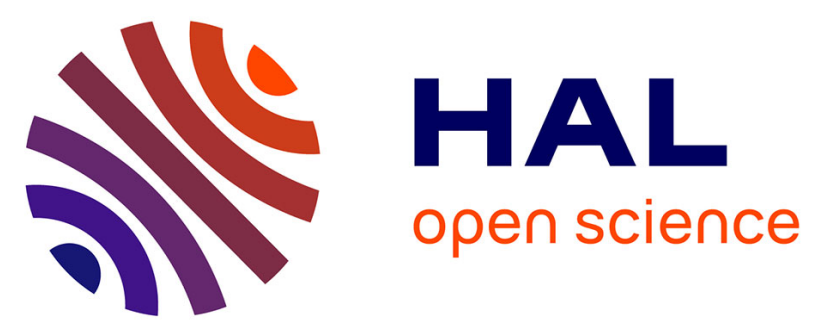

\title{
The binary systems associated with short and long gamma-ray bursts and their detectability
}

Jorge A. Rueda, Y. Aimuratov, U. Barres de Almeida, L. Becerra, C.L.

Bianco, C. Cherubini, S. Filippi, M. Karlica, M. Kovacevic, J.D. Melon

Fuksman, et al.

\section{To cite this version:}

Jorge A. Rueda, Y. Aimuratov, U. Barres de Almeida, L. Becerra, C.L. Bianco, et al.. The binary systems associated with short and long gamma-ray bursts and their detectability. Int.J.Mod.Phys.D, 2017, 26 (09), pp.1730016. 10.1142/S0218271817300166 . hal-01582797

\section{HAL Id: hal-01582797 \\ https://hal.science/hal-01582797}

Submitted on 13 Aug 2020

HAL is a multi-disciplinary open access archive for the deposit and dissemination of scientific research documents, whether they are published or not. The documents may come from teaching and research institutions in France or abroad, or from public or private research centers.
L'archive ouverte pluridisciplinaire HAL, est destinée au dépôt et à la diffusion de documents scientifiques de niveau recherche, publiés ou non, émanant des établissements d'enseignement et de recherche français ou étrangers, des laboratoires publics ou privés. 


\section{The binary systems associated with short and long gamma-ray bursts and their detectability*}

Jorge A. Rueda ${ }^{\dagger, \ddagger, \S, * * *}$, Y. Aimuratov ${ }^{\dagger, \ddagger, \uparrow}$, U. Barres de Almeidall,

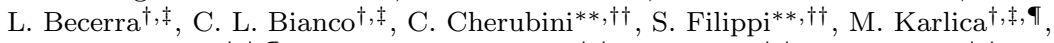
M. Kovacevic ${ }^{\dagger, \ddagger}$, , J. D. Melon Fuksman ${ }^{\dagger, \ddagger}$, R. Moradi ${ }^{\dagger, \ddagger}$, M. Muccino ${ }^{\dagger, \ddagger}$, A. V. Penacchioni ${ }^{\ddagger \ddagger}, \S \S$, G. B. Pisani ${ }^{\dagger, \ddagger}$, D. Primorac ${ }^{\dagger, \ddagger}$, R. Ruffini ${ }^{\dagger, \ddagger}, \S$, N. Sahakyan $₫$ ฯ, S. Shakeri ${ }^{\dagger},\|\|$ and Y. Wang ${ }^{\dagger, \ddagger}$

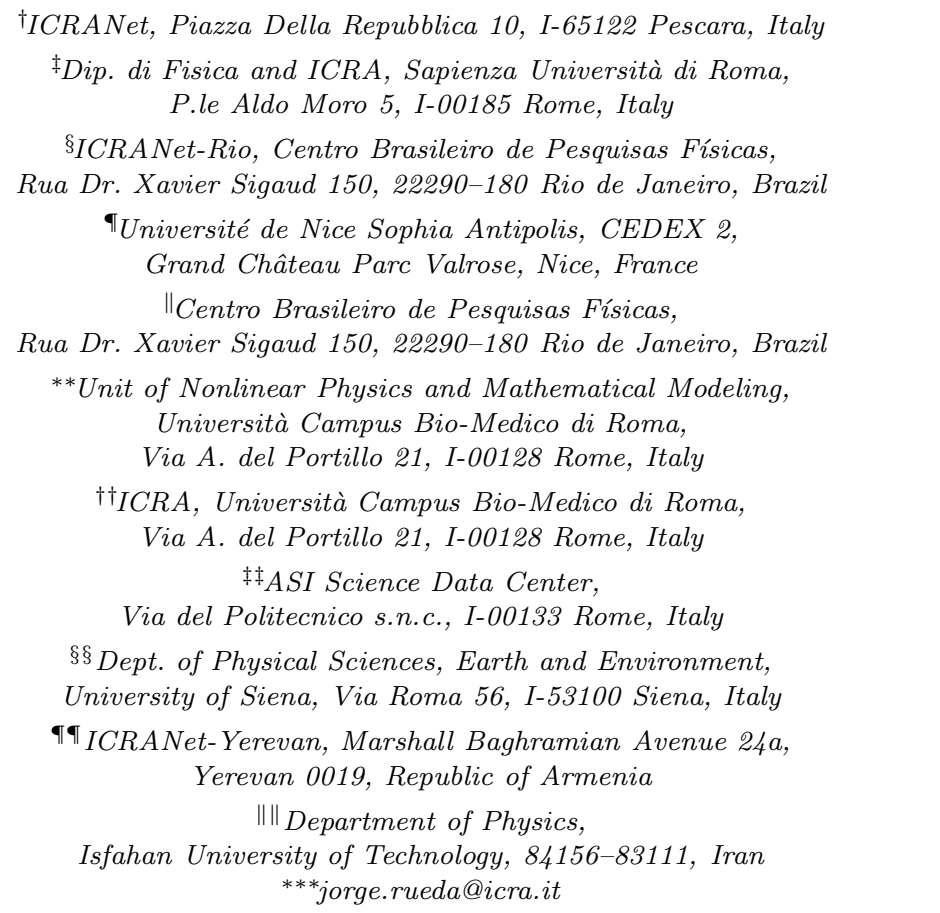

Received 2 March 2017

Accepted 3 March 2017

Published 13 April 2017

Short and long-duration gamma-ray bursts (GRBs) have been recently sub-classified into seven families according to the binary nature of their progenitors. For short GRBs, mergers of neutron star binaries (NS-NS) or neutron star-black hole binaries (NS-BH) are proposed. For long GRBs, the induced gravitational collapse (IGC) paradigm proposes

*Based on a talks presented at the Fourteenth Marcel Grossmann Meeting on General Relativity, Rome, July 2015. 
a tight binary system composed of a carbon-oxygen core $\left(\mathrm{CO}_{\text {core }}\right)$ and a NS companion. The explosion of the $\mathrm{CO}_{\text {core }}$ as supernova $(\mathrm{SN})$ triggers a hypercritical accretion process onto the NS companion which might reach the critical mass for the gravitational collapse to a BH. Thus, this process can lead either to a NS-BH or to NS-NS depending on whether or not the accretion is sufficient to induce the collapse of the NS into a BH. We shall discuss for the above compact object binaries: (1) the role of the NS structure and the equation-of-state on their final fate; (2) their occurrence rates as inferred from the X and gamma-ray observations; (3) the expected number of detections of their gravitational wave (GW) emission by the Advanced LIGO interferometer.

Keywords: Gamma-ray bursts; neutron stars; black holes.

PACS Number(s): 04.30.Tv, 04.40.Dg, 97.60.Bw, 97.60.Jd, 97.60.Lf, 98.62.Mw

\section{Introduction}

There has been a traditional phenomenological classification of GRBs based on the observed prompt duration, $T_{90}$ : long GRBs for $T_{90}>2 \mathrm{~s}$ and short GRBs for $T_{90}<2$ s. $^{1-5}$ In this paper, we shall review the recent progress reached in the understanding of the nature of long and short GRBs that has led to a physical GRB classification, proposed in Refs. 6-8. Such a classification, as we will see below, is based on the possible outcomes in the final stages of the evolution of the progenitor systems.

\subsection{Long GRBs}

The induced gravitational collapse (IGC) scenario introduces, as the progenitor of the long GRBs associated with $\mathrm{SNe} \mathrm{Ib} / \mathrm{c}$, binaries composed of a carbon-oxygen core $\left(\mathrm{CO}_{\text {core }}\right)$ on the verge of supernova with a NS companion. ${ }^{9-15}$ The explosion of the $\mathrm{CO}_{\text {core }}$ as $\mathrm{SN}$, forming at its center a newly-born NS called hereafter $\nu \mathrm{NS}$, triggers an accretion process onto the NS binary companion. Depending on the

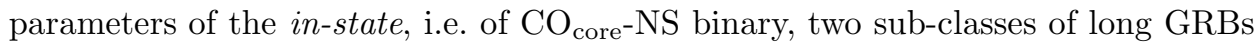
with corresponding out-states are envisaged ${ }^{6}$ :

- $X$-ray flashes $(X R F s)$. Long bursts with $E_{\text {iso }} \lesssim 10^{52} \mathrm{erg}$ are produced by $\mathrm{CO}_{\text {core }}{ }^{-}$ NS binaries with relatively large binary separations $\left(a \gtrsim 10^{11} \mathrm{~cm}\right)$. The accretion rate of the SN ejecta onto the NS in these systems is not high enough to bring the NS mass to the critical value $M_{\text {crit }}$, hence no $\mathrm{BH}$ is formed. The out-state of this GRB sub-class can be either a $\nu$ NS-NS binary if the system keeps bound after the SN explosion, or two runaway NSs if the binary system is disrupted.

- Binary driven hypernovae $(B d H N e)$. Long bursts with $E_{\text {iso }} \gtrsim 10^{52} \mathrm{erg}$ are instead produced by more compact $\mathrm{CO}_{\text {core }} \mathrm{NS}$ binaries $\left(a \lesssim 10^{11} \mathrm{~cm}\right.$, see e.g. Refs. 13 and 15$)$. In this case, the SN triggers a larger accretion rate onto the NS companion, e.g. $\gtrsim 10^{-2}-10^{-1} M_{\odot} \mathrm{s}^{-1}$, bringing the NS to its critical mass $M_{\text {crit }},{ }^{11-13}$ namely to the point of gravitational collapse with consequent formation of a $\mathrm{BH}$. Remarkably, in Ref. 14, it was recently shown that the large majority of BdHNe leads naturally to NS-BH binaries owing to the high compactness of the binary that avoids the disruption of it even in cases of very high mass loss exceeding $50 \%$ of the total mass of the initial $\mathrm{CO}_{\text {core }} \mathrm{NS}$ binary. 
In addition, it exists the possibility of BH-SNe. ${ }^{6}$ Long burst with $E_{\text {iso }} \gtrsim 10^{54} \mathrm{erg}$ occurring in close $\mathrm{CO}_{\text {core- }} \mathrm{BH}$ binaries in which the hypercritical accretion produces, as out-states, a more massive $\mathrm{BH}$ and a $\nu \mathrm{NS}$. These systems have been considered in Ref. 6 as a subset of the BdHNe but no specific example have been yet observationally identified.

\subsection{Short GRBs}

There is the consensus within the GRB community that the progenitors of short GRBs are mergers of NS-NS and/or NS-BH binaries (see, e.g. Refs. 16-20 for a recent review). Similarly to the case of long GRBs, in Ref. 6 short GRBs have been split into different sub-classes:

- Short gamma-ray flashes (S-GRFs). Short bursts with energies $E_{\text {iso }} \lesssim 10^{52} \mathrm{erg}$, produced when the post-merger core do not surpass the NS critical mass $M_{\text {crit }}$, hence there is no BH formation. Thus, these systems left as byproduct a massive NS and possibly, due to the energy and angular momentum conservation, orbiting material in a disk-like structure or a low-mass binary companion.

- Authentic short gamma-ray bursts $(S-G R B s)$. Short bursts with $E_{\text {iso }} \gtrsim 10^{52} \mathrm{erg}$, produced when the post-merger core reaches or overcome $M_{\text {crit }}$, hence forming a Kerr or Kerr-Newman $\mathrm{BH},{ }^{8}$ and also in this case possibly orbiting material.

- Ultra-short GRBs (U-GRBs). A new sub-class of short bursts originating from $\nu \mathrm{NS}-\mathrm{BH}$ merging binaries. They can originate from BdHNe (see Ref. 14) or from $\mathrm{BH}-\mathrm{SNe}$.

In addition, it exists the possibility of gamma-ray flashes (GRFs). These are bursts with hybrid properties between short and long, they have $10^{51} \lesssim E_{\text {iso }} \lesssim$ $10^{52} \mathrm{erg}$. This sub-class of sources originates in NS-WD mergers. ${ }^{6}$

Table 1 summarized some observational aspects of the GRB sub-classes including the occurrence rate calculated in Ref. 6 .

Table 1. Some observational aspects of the GRB sub-classes. In the first three columns, we indicate the GRB sub-class and their corresponding in-states and the out-states. In column 4, we list the $E_{\text {iso }}($ rest-frame 1-10 $\mathrm{keV}$ ), columns 5-6 list, for each GRB sub-class, the maximum observed redshift and the local occurrence rate computed in Ref. 6 .

\begin{tabular}{lccccc}
\hline GRB sub-class & In-state & Out-state & $\begin{array}{c}E_{\text {iso }} \\
(\mathrm{erg})\end{array}$ & $z_{\max }$ & $\begin{array}{c}\rho_{\mathrm{GRB}} \\
\left(\mathrm{Gpc}^{-3} \mathrm{yr}^{-1}\right)\end{array}$ \\
\hline XRFs & $\mathrm{CO}_{\text {core-NS }}$ & $\nu \mathrm{NS}-\mathrm{NS}$ & $10^{48}-10^{52}$ & 1.096 & $100_{-34}^{+45}$ \\
BdHNe & $\mathrm{CO}_{\text {core }}$-NS & $\nu \mathrm{NS}-\mathrm{BH}$ & $10^{52}-10^{54}$ & 9.3 & $0.77_{-0.08}^{+0.09}$ \\
BH-SN & $\mathrm{CO}_{\text {core- }}$ BH & $\nu \mathrm{NS}-\mathrm{BH}$ & $>10^{54}$ & 9.3 & $\lesssim 0.77_{-0.08}^{+0.09}$ \\
S-GRFs & $\mathrm{NS}-\mathrm{NS}$ & $\mathrm{MNS}$ & $10^{49}-10^{52}$ & 2.609 & $3.6_{-1.0}^{+1.4}$ \\
S-GRBs & $\mathrm{NS}-\mathrm{NS}$ & $\mathrm{BH}$ & $10^{52}-10^{53}$ & 5.52 & $\left(1.9_{-1.1}^{+1.8}\right) \times 10^{-3}$ \\
U-GRBs & $\nu \mathrm{NS}-\mathrm{BH}$ & $\mathrm{BH}$ & $>10^{52}$ & - & $\gtrsim 0.77_{-0.08}^{+0.09}$ \\
GRFs & $\mathrm{NS}-\mathrm{WD}$ & $\mathrm{MNS}$ & $10^{51}-10^{52}$ & 2.31 & $1.02_{-0.46}^{+0.71}$ \\
\hline
\end{tabular}


We focus here on the physical properties of the above progenitors, as well as on the main properties of NSs that play a relevant role in the dynamics of these systems and that lead to the above different GRB sub-classes. We shall discuss as well recent estimates of the rates of occurrence on all the above subclasses based on $\mathrm{X}$ and gamma-ray observations, and also elaborate on the possibility of detecting the gravitational wave (GW) emission originated in these systems.

\section{IGC, Hypercritical Accretion, and Long GRBs}

We turn now to the details of the accretion process within the IGC scenario. Realistic simulations of the IGC process were performed in Ref. 12, including: (1) detailed $\mathrm{SN}$ explosions of the $\mathrm{CO}_{\text {core }}$; (2) the hydrodynamic details of the hypercritical accretion process; (3) the evolution of the SN ejecta material entering the Bondi-Hoyle region all the way up to its incorporation into the NS. Here, the concept of hypercritical accretion refers to the fact the accretion rates are highly super-Eddington. The accretion process in the IGC scenario is allowed to exceed the Eddington limit mainly for two reasons: (i) the photons are trapped within the infalling material impeding them to transfer momentum; (ii) the accreting material creates a very hot NS atmosphere $\left(T \sim 10^{10} \mathrm{~K}\right)$ that triggers a very efficient neutrino emission which become the main energy sink of these systems unlike photons.

The hypercritical accretion process in the above simulations was computed within a spherically symmetric approximation. A further step was given in Ref. 13 by estimating the angular momentum that the SN ejecta carries and transfer to the NS via accretion, and how it affects the evolution and fate of the system. The calculations are as follows: first the accretion rate onto the NS is computed adopting an homologous expansion of the SN ejecta and introducing the pre-SN density profile of the $\mathrm{CO}_{\text {core }}$ envelope from numerical simulations. Then, it is estimated the angular momentum that the SN material might transfer to the NS: it comes out that the ejecta have enough angular momentum to circularize for a short time and form a disc around the NS. Finally, the evolution of the NS central density and rotation angular velocity (spin-up) is followed computing the equilibrium configurations from the numerical solution of the axisymmetric Einstein equations in full rotation, until the critical point of collapse of the NS to a BH taking into due account the equilibrium limits given by mass-shedding and the secular axisymmetric instability.

Now we enter into the details of each of the above steps. The accretion rate of the SN ejecta onto the NS can be estimated via the Bondi-Hoyle-Lyttleton accretion formula:

$$
\dot{M}_{B}(t)=\pi \rho_{\text {ej }} R_{\text {cap }}^{2} \sqrt{v_{\text {rel }}^{2}+c_{\mathrm{s}, \mathrm{ej}}^{2}}, \quad R_{\mathrm{cap}}(t)=\frac{2 G M_{\mathrm{NS}}(t)}{v_{\mathrm{rel}}^{2}+c_{\mathrm{s}, \mathrm{ej}}^{2}},
$$

where $G$ is the gravitational constant, $\rho_{\mathrm{ej}}$ and $c_{\mathrm{s}, \mathrm{ej}}$ are the density and sound speed of the SN ejecta, $R_{\text {cap }}$ is the NS gravitational capture radius (Bondi-Hoyle radius), $M_{\mathrm{NS}}$, the NS mass, and $v_{\mathrm{rel}}$ the ejecta velocity relative to the NS: $\mathbf{v}_{\mathrm{rel}}=\mathbf{v}_{\mathrm{orb}}-\mathbf{v}_{\mathrm{ej}}$, 


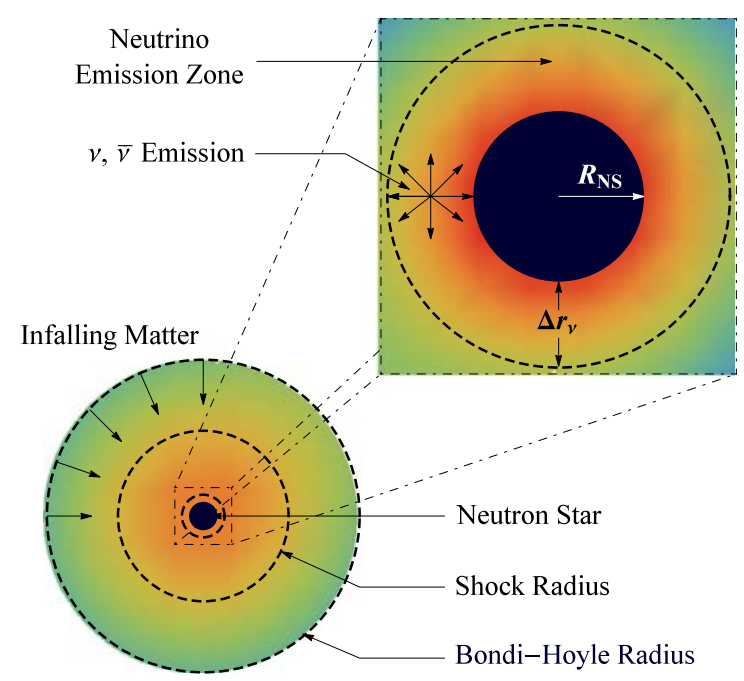

Fig. 1. Scheme of the IGC scenario: the $\mathrm{CO}_{\text {core }}$ undergoes SN explosion, the NS accretes part of the SN ejecta and then reaches the critical mass for gravitational collapse to a $\mathrm{BH}$, with consequent emission of a GRB. The SN ejecta reach the NS Bondi-Hoyle radius and fall toward the NS surface. The material shocks and decelerates as it piles over the NS surface. At the neutrino emission zone, neutrinos take away most of the infalling matter gravitational energy gain. The neutrinos are emitted above the NS surface in a region of thickness $\Delta r_{\nu}$ about half the NS radius that allow the material to reduce its entropy to be finally incorporated to the NS. The image is not to scale. For further details and numerical simulations of the above process, see Refs. 12-15.

with $\left|\mathbf{v}_{\text {orb }}\right|=\sqrt{G\left(M_{\text {core }}+M_{\mathrm{NS}}\right) / a}$, the module of the NS orbital velocity around the $\mathrm{CO}_{\text {core }}$, and $\mathbf{v}_{\mathrm{ej}}$ the velocity of the supernova ejecta (see Fig. 1).

Extrapolating the results for the accretion process from stellar wind accretion in binary systems, the angular momentum per unit time that crosses the NS capture region can be approximated by: $\dot{L}_{\text {cap }}=(\pi / 2)\left(\epsilon_{\rho} / 2-3 \epsilon_{\nu}\right) \rho_{\text {ej }}(a, t) v_{\text {rel }}^{2}(a, t) R_{\text {cap }}^{4}(a, t)$, where $\epsilon_{\rho}$ and $\epsilon_{\nu}$ are parameters measuring the inhomogeneity of the flow (see Ref. 13 for details).

In order to simulate the hypercritical accretion, it is adopted an homologous expansion of the $\mathrm{SN}$ ejecta, i.e. the ejecta velocity evolves as $v_{\mathrm{ej}}(r, t)=$ $n r / t$, where $r$ is the position of every ejecta layer from the SN center and $n$ is called expansion parameter. The ejecta density is given by $\rho_{\mathrm{ej}}(r, t)=$ $\rho_{\text {ej }}^{0}\left(r / R_{\text {star }}(t), t_{0}\right) \frac{M_{\text {env }}(t)}{M_{\text {env }}(0)}\left(\frac{R_{\text {star }}(0)}{R_{\text {star }}(t)}\right)^{3}$, where $M_{\text {env }}(t)$ the mass of the $\mathrm{CO}_{\text {core }}$ envelope, namely the mass of the ejected material in the SN explosion and available to be accreted by the NS, $R_{\text {star }}(t)$ is the position of the outermost layer of the ejected material, and $\rho_{\text {ej }}^{0}$ is the pre-SN density profile. The latter can be approximated with a power law: $\rho_{\mathrm{ej}}\left(r, t_{0}\right)=\rho_{\text {core }}\left(R_{\text {core }} / r\right)^{m}$, where $\rho_{\text {core }}, R_{\text {core }}$ and $m$ are the profile parameters which are fixed by fitting the pre-SN profiles obtained from numerical simulations.

For the typical parameters of pre-SN $\mathrm{CO}_{\text {core }}$ and assuming a velocity of the outermost SN layer $v_{\mathrm{sn}}\left(R_{\mathrm{star}}, t_{0}\right) \sim 10^{9} \mathrm{~cm} \mathrm{~s}^{-1}$ and a free expansion $n=1$ (for details 
of typical initial conditions of the binary system see Refs. 12 and 13), Eq. (1) gives accretion rates around the order of $10^{-4}-10^{-2} M_{\odot} \mathrm{s}^{-1}$, and an angular momentum per unit time crossing the capture region $\dot{L}_{\text {cap }} \sim 10^{46}-10^{49} \mathrm{~g} \mathrm{~cm}^{2} \mathrm{~s}^{-2}$.

We consider the NS companion of the $\mathrm{CO}_{\text {core }}$ initially as nonrotating, thus at the beginning, the NS exterior spacetime is described by the Schwarzschild metric. The SN ejecta approach the NS with specific angular momentum, $l_{\text {acc }}=\dot{L}_{\text {cap }} / \dot{M}_{B}$, thus they will circularize at a radius $r_{\text {st }}$ if they have enough angular momentum. What does the word "enough" means here? The last stable circular orbit (LSO) around a nonrotating NS is located at a distance $r_{\mathrm{lso}}=6 G M_{\mathrm{NS}} / c^{2}$ and has an angular momentum per unit mass $l_{\mathrm{lso}}=2 \sqrt{3} G M_{\mathrm{NS}} / c$. The radius $r_{\mathrm{lso}}$ is larger than the NS radius for masses larger than $1.67 M_{\odot}, 1.71 M_{\odot}$, and $1.78 M_{\odot}$ for the GM1, TM1, and NL3 nuclear equation-of-state (EOS). ${ }^{13}$ If $l_{\text {acc }} \geq l_{\text {lso }}$, the material circularizes around the NS at locations $r_{\text {st }} \geq r_{\text {lso }}$. For the values of the IGC systems under discussion here, $r_{\text {st }} / r_{\text {lso }} \sim 10-10^{3}$, thus the SN ejecta have enough angular momentum to form a sort of disc around the NS. Even in this case, the viscous forces and other angular momentum losses that act on the disk will allow the matter in the disk to reach the inner boundary at $r_{\text {in }} \sim r_{\text {lso }}$, to then be accreted by the NS.

Within this picture, the NS accretes the material from $r_{\text {in }}$ and the NS mass and angular momentum evolve as:

$$
\dot{M}_{\mathrm{NS}}=\left(\frac{\partial M_{\mathrm{NS}}}{\partial M_{b}}\right)_{J_{\mathrm{NS}}} \dot{M}_{b}+\left(\frac{\partial M_{\mathrm{NS}}}{\partial J_{\mathrm{NS}}}\right)_{M_{b}} \dot{J}_{\mathrm{NS}}, \quad \dot{J}_{\mathrm{NS}}=\xi l\left(r_{\mathrm{in}}\right) \dot{M}_{\mathrm{B}},
$$

where $M_{b}$ is the NS baryonic mass, $l\left(r_{\text {in }}\right)$ is the specific angular momentum of the accreted material at $r_{\text {in }}$, which corresponds to the angular momentum of the LSO, and $\xi \leq 1$ is a parameter that measures the efficiency of angular momentum transfer. We assume in our simulations $\dot{M}_{b}=\dot{M}_{B}$.

In order to integrate Eqs. (1) and (2), we have to supply the two above partial derivatives which are obtained from the relation of the NS gravitational mass with $M_{b}$ and $J_{\mathrm{NS}}$, namely from the NS binding energy. The general relativistic calculations of rotating NSs in Ref. 21 show that, independent on the nuclear EOS, this relation is well approximated by the formula

$$
\frac{M_{b}}{M_{\odot}}=\frac{M_{\mathrm{NS}}}{M_{\odot}}+\frac{13}{200}\left(\frac{M_{\mathrm{NS}}}{M_{\odot}}\right)^{2}\left(1-\frac{1}{137} j_{\mathrm{NS}}^{1.7}\right),
$$

where $j_{\mathrm{NS}} \equiv c J_{\mathrm{NS}} /\left(G M_{\odot}^{2}\right)$. In addition, since the NS will spin up with accretion, we need information of the dependence of the specific angular momentum of the LSO as a function of both the NS mass and angular momentum. For corotating orbits, the following relation is valid for all the aforementioned $\operatorname{EOS}^{13}$ :

$$
l_{\mathrm{lso}}=\frac{G M_{\mathrm{NS}}}{c}\left[2 \sqrt{3}-0.37\left(\frac{\frac{j_{\mathrm{NS}}}{M_{\mathrm{NS}}}}{M_{\odot}}\right)^{0.85}\right] .
$$

The NS accretes mass until it reaches a region of instability. There are two main instability limits for rotating NSs: mass-shedding or Keplerian limit and the secular 
Table 2. Critical NS mass in the nonrotating case and constants $k$ and $p$ needed to compute the NS critical mass in the nonrotating case given by Eq. (5). The values are given for the NL3, GM1 and TM1 EOS.

\begin{tabular}{lccc}
\hline EOS & $M_{\text {crit }}^{J=0}\left(M_{\odot}\right)$ & $p$ & $k$ \\
\hline NL3 & 2.81 & 1.68 & 0.006 \\
GM1 & 2.39 & 1.69 & 0.011 \\
TM1 & 2.20 & 1.61 & 0.017 \\
\hline
\end{tabular}

axisymmetric instability. The critical NS mass along the secular instability line is approximately given $b^{21}$ :

$$
M_{\mathrm{NS}}^{\mathrm{crit}}=M_{\mathrm{NS}}^{J=0}\left(1+k j_{\mathrm{NS}}^{p}\right)
$$

where the parameters $k$ and $p$ depends of the nuclear EOS (see Table 2). These formulas fit the numerical results with a maximum error of $0.45 \%$.

Along the mass-shedding sequence, the NS has the maximum possible angular momentum ${ }^{21}: J_{\mathrm{NS}, \max } \approx 0.7 G M_{\mathrm{NS}}^{2} / c$. Figure 2 shows the evolution of the NS dimensionless angular momentum, $c J_{\mathrm{NS}} /\left(G M_{\mathrm{NS}}^{2}\right)$, as a function of the NS mass for $\xi=0.5$ and for selected values of the initial NS mass. The NS fate depends of the NS initial mass and the efficiency parameter $\xi$. The less massive initial configurations reach the mass-shedding limit with a maximum dimensionless angular momentum value while the initially more massive configurations reach the secular axisymmetric instability. It is interesting to note that the total angular momentum of the SN ejecta entering the Bondi-Hoyle region, $L_{\text {cap }}$, is much larger than the

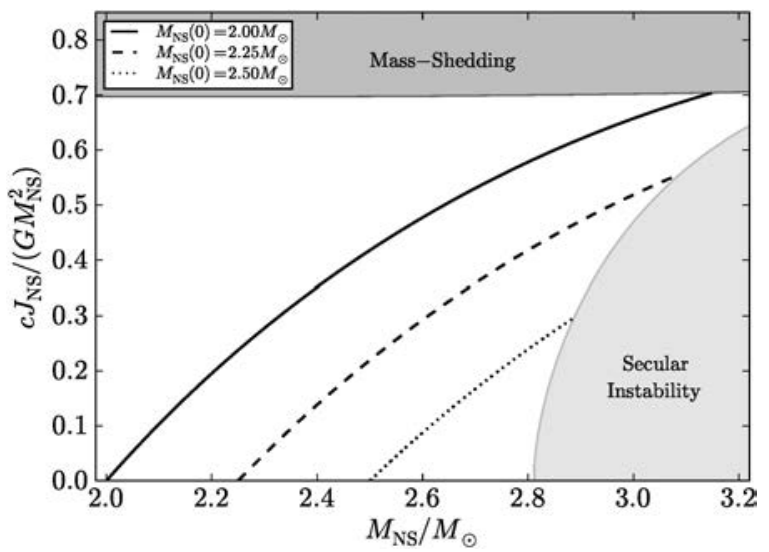

Fig. 2. Evolution of NSs of different initial masses $M_{\mathrm{NS}}=2.0,2.25$ and $2.5 M_{\odot}$ during the hypercritical accretion in a BdHN. ${ }^{13}$ It is shown the dimensionless angular momentum as a function of the NS mass. The binary parameters are: $\mathrm{CO}_{\text {core }}$ of a $M_{\mathrm{ZAMS}}=30 M_{\odot}$ progenitor star $\left(m=2.801, M_{\text {env }}=7.94 M_{\odot}, \rho_{\text {core }}=3.08 \times 10^{8} \mathrm{~g} \mathrm{~cm}^{-3}\right.$ and $\left.R_{0_{\text {star }}}=7.65 \times 10^{9} \mathrm{~cm}\right)$, a free expansion $(n=1)$ and a SN outermost ejecta velocity $v_{0_{\text {star }}}=2 \times 10^{9} \mathrm{~cm} \mathrm{~s}^{-1}$. The orbital period is of approximately $5 \mathrm{~min}$. 
maximum angular momentum that a uniformly rotating NS can support, $J_{\mathrm{NS} \text {, max }}$. The numerical simulations in Ref. 13 indicate $L_{\text {cap }} \sim 10 J_{\mathrm{NS} \text {, max }}$. Thus, part of this angular momentum must be lost or redistributed before the material can reach the NS surface. This result leads to a clear prediction: the BHs produced through the IGC mechanism, namely those formed in BdHNe, have initial dimensionless spin $\sim 0.7$ and the excess of angular momentum could lead to a jetted emission with possible high-energy signatures and/or to the presence of a disk-like structure first around the NS as shown above and possibly also around the $\mathrm{BH}$ originated from the gravitational collapse of the NS.

\subsection{Most recent simulations of the IGC process}

Additional details and improvements of the hypercritical accretion process leading to XRFs and BdHNe have been recently presented in Ref. 15. In particular:

(1) It was there improved the accretion rate estimate including the density profile finite size/thickness and additional $\mathrm{CO}_{\text {core }}$ progenitors leading to different SN ejecta masses were also considered.

(2) It was shown in Ref. 13, the existence of a maximum orbital period, $P_{\max }$, over which the accretion onto NS companion is not high enough to bring it to the critical mass for gravitational collapse to a $\mathrm{BH}$. Therefore, $\mathrm{CO}_{\text {core }}-\mathrm{NS}$ binaries with $P>P_{\max }$ lead to XRFs while the ones with $P \lesssim P_{\max }$ lead to BdHNe. In Ref. 15, the determination of $P_{\max }$ was extended to all the possible initial values of the mass of the NS companion and the angular momentum transfer efficiency parameter was also allowed to vary.

(3) It was computed the expected luminosity during the hypercritical accretion process for a wide range of binary periods covering XRFs and BdHNe.

(4) It was there shown that the presence of the NS companion originates large asymmetries (see, e.g. simulation in Fig. 3) in the SN ejecta leading to observable signatures in the X-rays.

Figure 3 shows a simulation of an IGC process presented in Ref. 15. We considered the effects of the gravitational field of the NS on the SN ejecta including the orbital motion as well as the changes in the NS gravitational mass owing to the accretion process via the Bondi formalism. The supernova matter was described as formed by point-like particles whose trajectory was computed by solving the Newtonian equation of motion. The initial conditions of the SN ejecta are computed assuming an homologous velocity distribution in free expansion. The initial powerlaw density profile of the $\mathrm{CO}$ envelope is simulated by populating the inner layers with more particles. For the $M_{\mathrm{ZAMS}}=30 M_{\odot}$ progenitor which gives a $\mathrm{CO}_{\text {core }}$ with envelope profile $\rho_{\mathrm{ej}}^{0} \approx 3.1 \times 10^{8}\left(8.3 \times 10^{7} / r\right)^{2.8} \mathrm{~g} \mathrm{~cm}^{-3}$, we adopt for the simulation a total number of $N=10^{6}$ particles. We assume that particles crossing the Bondi-Hoyle radius are captured and accreted by the NS so we removed them from the system as they reach that region. We removed these particles according to the 

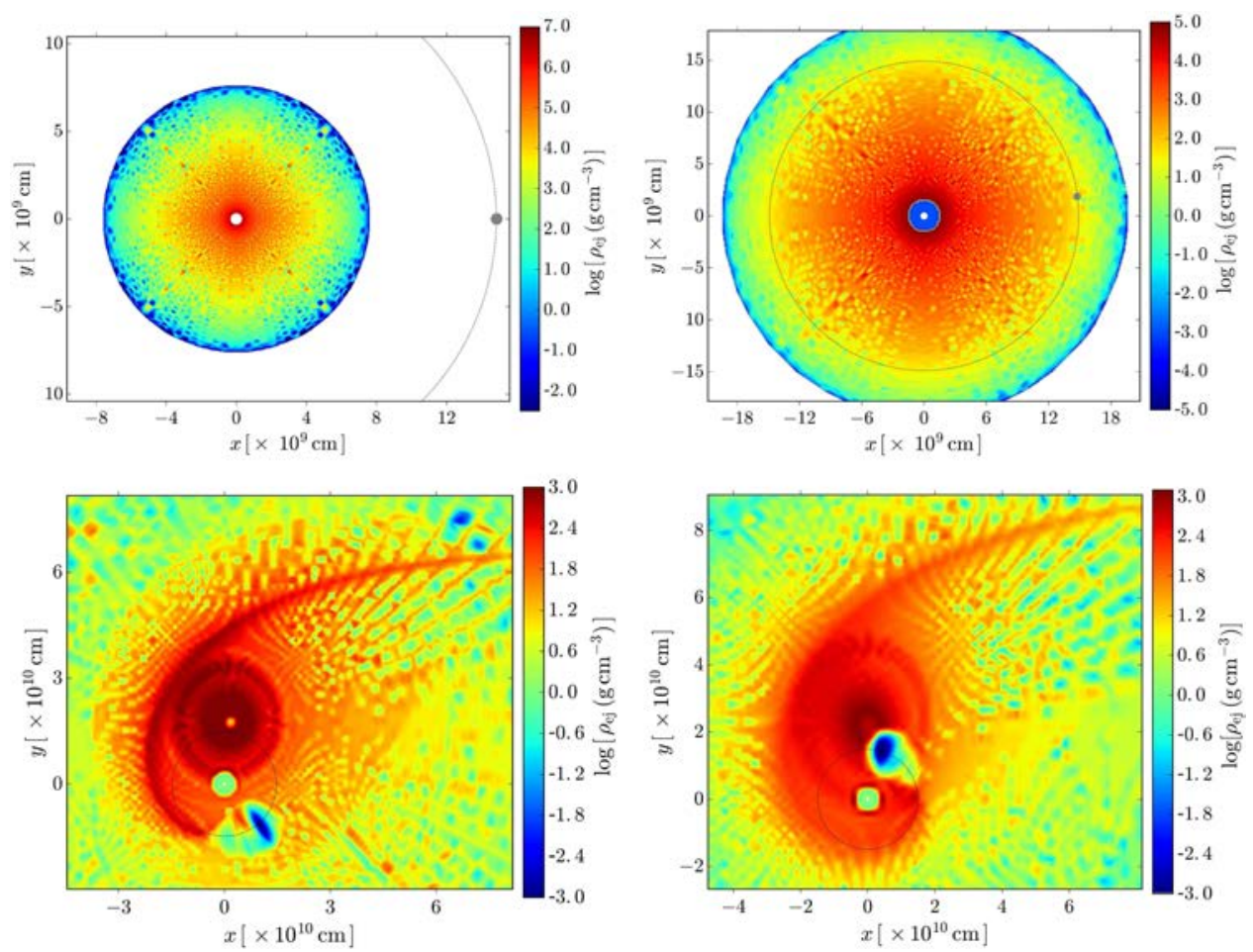

Fig. 3. Hypercritical accretion process in the IGC binary system at selected evolution times. In this example, the $\mathrm{CO}_{\text {core }}$ has a total mass of $9.44 M_{\odot}$ divided in an ejecta mass of $7.94 M_{\odot}$ and a $\nu \mathrm{NS}$ of $1.5 \mathrm{M}_{\odot}$ formed by the collapsed high density core. The supernova ejecta evolve homologously with outermost layer velocity $v_{0, \text { star }}=2 \times 10^{9} \mathrm{~cm} \mathrm{~s}^{-1}$. The NS binary companion has an initial mass of $2.0 \mathrm{M}_{\odot}$. The binary period is $P \approx 5 \mathrm{~min}$, which corresponds to a binary separation $a \approx 1.5 \times 10^{10} \mathrm{~cm}$. The system of coordinates is centered on the $\nu$ NS represented by the white-filled circle at $(0,0)$. The NS binary companion, represented by the gray-filled circle, orbits counterclockwise following the thin-dashed circular trajectory. The colorbar indicates values of ejecta density in logarithmic scale. Left upper panel: initial time of the process. The supernova ejecta expand radially outward and the NS binary companion is at $(a, 0)$. Right upper panel: the accretion process starts when the first supernova layers reach the Bondi-Hoyle region. This happens at $t=t_{\mathrm{acc}, 0} \approx a / v_{0, \text { star }} \approx 7.7 \mathrm{~s}$. Left lower panel: the NS binary companion reaches the critical mass by accreting matter from the SN with consequent collapse to a $\mathrm{BH}$. This happens at $t=t_{\text {coll }} \approx 254 \mathrm{~s} \approx 0.85 P$. The newly-formed BH of mass $M_{\mathrm{BH}}=M_{\text {crit }} \approx 3 M_{\odot}$ is represented by the black-filled circle. It is here evident the asymmetry of the supernova ejecta induced by the presence of the accreting NS companion at close distance. Right lower panel: $t=t_{\text {coll }}+100 \mathrm{~s}=$ $354 \mathrm{~s} \approx 1.2 P$, namely $100 \mathrm{~s}$ after the $\mathrm{BH}$ formation. It appears here the new binary system composed of the $\nu \mathrm{NS}$ and the newly-formed $\mathrm{BH}$.

results obtained from the numerical integration explained above. Figure 3 shows the orbital plane of an IGC binary at selected times of its evolution. The NS has an initial mass of $2.0 M_{\odot}$; the $\mathrm{CO}_{\text {core }}$ leads to a total ejecta mass $7.94 M_{\odot}$ and a $\nu \mathrm{NS}$ of $1.5 M_{\odot}$. The orbital period of the binary is $P \approx 5 \mathrm{~min}$, i.e. a binary separation $a \approx 1.5 \times 10^{10} \mathrm{~cm}$. For these parameters, the NS reaches the critical mass and collapses to form a $\mathrm{BH}$. 


\subsection{Hydrodynamics and neutrino inside the accretion region}

We turn now to give some details on the properties of the system inside the BondiHoyle accretion region. We have seen that the accretion rate onto the NS can be as high as $\sim 10^{-2}-10^{-1} M_{\odot} \mathrm{s}^{-1}$. For these accretion rates:

(1) We can neglect the effect of the NS magnetic field since the magnetic pressure remains much smaller than the random pressure of the infalling material. ${ }^{11,22}$

(2) The photons are trapped in the accretion flow. The trapping radius, defined at which the photons emitted diffuse outward at a slower velocity than the one of the infalling material, is ${ }^{23}: r_{\text {trapping }}=\min \left\{\dot{M}_{B} \kappa /(4 \pi c), R_{\text {cap }}\right.$, where $\kappa$ is the opacity. For the $\mathrm{CO}_{\text {core, }}$ in Ref. 12, a Rosseland mean opacity roughly $5 \times 10^{3} \mathrm{~cm}^{2} \mathrm{~g}^{-1}$ was estimated. For the range of accretion rates, we obtain that $\dot{M}_{B} \kappa /(4 \pi c) \sim 10^{13}-10^{19} \mathrm{~cm}$, a radius much bigger than the NS capture radius which is in our simulations at most $1 / 3$ of the binary separation. Thus, in our systems, the trapping radius extends all the way to the Bondi-Hoyle region, hence the Eddington limit does not apply and hypercritical accretion onto the NS occurs.

(3) Under these conditions, the gain of gravitational energy of the accreted material is mainly radiated via neutrino emission (see below). ${ }^{11,12,22,24,25}$

\subsubsection{Convective instabilities}

As the material piles onto the NS and the atmosphere radius, the accretion shock moves outward. The post-shock entropy is a decreasing function of the shock radius position which creates an atmosphere unstable to Rayleigh-Taylor convection during the initial phase of the accretion process. These instabilities can accelerate above the escape velocity driving outflows from the accreting NS with final velocities approaching the speed of light. ${ }^{26,27}$ Assuming that radiation dominates, the entropy of the material at the base of the atmosphere is ${ }^{22}: S_{\text {bubble }} \approx$ $16\left(1.4 M_{\odot} / M_{\mathrm{NS}}\right)^{-7 / 8}\left(M_{\odot} \mathrm{s}^{-1} / \dot{M}_{\mathrm{B}}\right)^{1 / 4}\left(10^{6} \mathrm{~cm} / r\right)^{3 / 8}$, in units of $k_{B}$ per nucleon. This material will rise and expand, cooling adiabatically, i.e. $T^{3} / \rho=$ constant, for radiation dominated gas. If we assume a spherically symmetric expansion, then $\rho \propto 1 / r^{3}$ and we obtain $k_{B} T_{\text {bubble }}=195 S_{\text {bubble }}^{-1}\left(10^{6} \mathrm{~cm} / r\right) \mathrm{MeV}$. However, it is more likely that the bubbles expand in the lateral but not in the radial direction, ${ }^{27}$ thus we have $\rho \propto 1 / r^{2}$, i.e. $T_{\text {bubble }}=T_{0}\left(S_{\text {bubble }}\right)\left(r_{0} / r\right)^{2 / 3}$, where $T_{0}\left(S_{\text {bubble }}\right)$ is given by the above equation evaluated at $r=r_{0} \approx R_{\mathrm{NS}}$. This temperature implies a bolometric blackbody flux at the source from the bubbles

$$
\begin{aligned}
F_{\text {bubble }} \approx & 2 \times 10^{40}\left(\frac{M_{\mathrm{NS}}}{1.4 M_{\odot}}\right)^{-7 / 2}\left(\frac{\dot{M}_{\mathrm{B}}}{M_{\odot} \mathrm{s}^{-1}}\right)\left(\frac{R_{\mathrm{NS}}}{10^{6} \mathrm{~cm}}\right)^{3 / 2} \\
& \times\left(\frac{r_{0}}{r}\right)^{8 / 3} \operatorname{erg~s}^{-1} \mathrm{~cm}^{-2},
\end{aligned}
$$

where $\sigma$ is the Stefan-Boltzmann constant. 
In Ref. 12, it was shown that the above thermal emission from the rising bubbles produced during the hypercritical accretion process can explain the early $(t \lesssim 50 \mathrm{~s})$ thermal X-ray emission observed in GRB 090618. ${ }^{10,28}$ In that case, $T_{\text {bubble }}$ drops from $50 \mathrm{keV}$ to $15 \mathrm{keV}$ expanding from $r \approx 10^{9} \mathrm{~cm}$ to $6 \times 10^{9} \mathrm{~cm}$, for an accretion rate $10^{-2} M_{\odot} \mathrm{s}^{-1}$.

It is interesting that also r-process nucleosynthesis can occur in these outflows. ${ }^{26}$ This implies that long GRBs can be also r-process sites with specific signatures from the decay of the produced heavy elements, possibly similar as in the case of the kilonova emission in short GRBs. ${ }^{29}$ The signatures of this phenomenon in XRFs and BdHNe, and its comparison with kilonovae, deserves to be explored.

\subsubsection{Neutrino emission}

Most of the energy from the accretion is lost through neutrino emission. For the accretion rate conditions characteristic of our models $\sim 10^{-4}-10^{-2} M_{\odot} \mathrm{s}^{-1}, e^{+} e^{-}$ pair annihilation dominates the neutrino emission and electron neutrinos remove the bulk of the energy. The temperature of these neutrinos can be roughly approximated by assuming that the inflowing material generally flows near to the NS surface before shocking and emitting neutrinos. For accretion rates $\sim 10^{-4}-10^{-2} M_{\odot} \mathrm{s}^{-1}$, neutrino energies $\sim 5-20 \mathrm{MeV}$ are obtained. ${ }^{15}$ A detailed study of the neutrino emission will be the presented elsewhere.

For the developed temperatures (say $k_{B} T \sim 1-10 \mathrm{MeV}$ ) near the NS surface, the dominant neutrino emission process is the $e^{+} e^{-}$annihilation leading to $\nu \bar{\nu}$. This process produces a neutrino emissivity proportional to the ninth power of the temperature. The accretion atmosphere near the NS surface is characterized by a temperature gradient with a typical scale height $\Delta r_{\nu} \approx 0.7 R_{\mathrm{NS}} \cdot{ }^{15}$ Owing to the aforementioned strong dependence of the neutrino emission on temperature, most of the neutrino emission occurs in the region $\Delta r_{\nu}$ above the NS surface.

These conditions lead to the neutrinos to be efficient in balancing the gravitational potential energy gain allowing the hypercritical accretion rates. The effective accretion onto the NS can be estimated $\mathrm{as}^{22}: \dot{M}_{\text {eff }} \approx \Delta M_{\nu}\left(L_{\nu} / E_{\nu}\right)$, where $\Delta M_{\nu}$ and $L_{\nu}$ are the mass and neutrino luminosity in the emission region (i.e. $\Delta r_{\nu}$ ). $E_{\nu}$ is half the gravitational potential energy gained by the material falling from infinity to the $R_{\mathrm{NS}}+\Delta r_{\nu}$. Since $L_{\nu} \approx 2 \pi R_{\mathrm{NS}}^{2} \Delta r_{\nu} \epsilon_{\mathrm{e}^{-} \mathrm{e}^{+}}$with $\epsilon_{\mathrm{e}^{-} \mathrm{e}^{+}}$the $e^{+} e^{-}$pair annihilation process emissivity, and $E_{\nu}=(1 / 2) G M_{\mathrm{NS}} \Delta M_{\nu} /\left(R_{\mathrm{NS}}+\Delta r_{\nu}\right)$, it can be checked that for $M_{\mathrm{NS}}=1.4 M_{\odot}$ this accretion rate leads to values $\dot{M}_{\mathrm{eff}} \approx 10^{-9}-10^{-1} M_{\odot} \mathrm{s}^{-1}$ for temperatures $k_{B} T=1-10 \mathrm{MeV}$.

\subsection{Accretion luminosity}

The gain of gravitational potential energy in the accretion process is the total one available to be released, e.g. by neutrinos and photons. The total energy released in the star in a time-interval $d t$ during the accretion of an amount of mass $d M_{b}$ with 
angular momentum $l \dot{M}_{b}$, is given by ${ }^{13,30}$

$$
L_{\mathrm{acc}}=\left(\dot{M}_{b}-\dot{M}_{\mathrm{NS}}\right) c^{2}=\dot{M}_{b} c^{2}\left[1-\left(\frac{\partial M_{\mathrm{NS}}}{\partial J_{\mathrm{NS}}}\right)_{M_{b}} l-\left(\frac{\partial M_{\mathrm{NS}}}{\partial M_{b}}\right)_{J_{\mathrm{NS}}}\right]
$$

This upper limit to the energy released is just the amount of gravitational energy gained by the accreted matter by falling to the NS surface and which is not spent in changing the gravitational binding energy of the NS. The total energy releasable during the accretion process, say $\Delta E_{\mathrm{acc}} \equiv \int L_{\mathrm{acc}} d t$, is given by the difference in binding energies of the initial and final NS configurations. The typical luminosity will be $L_{\text {acc }} \approx \Delta E_{\text {acc }} / \Delta t_{\text {acc }}$ where $\Delta t_{\text {acc }}$ is the duration of the accretion process.

The duration of the accretion process is given approximately by the flow time of the slowest layers of the supernova ejecta to the NS. If the velocity of these layers is $v_{\text {inner }}$, then $\Delta t_{\text {acc }} \sim a / v_{\text {inner }}$, where $a$ is the binary separation. For $a \sim 10^{11} \mathrm{~cm}$ and $v_{\text {inner }} \sim 10^{8} \mathrm{~cm} \mathrm{~s}^{-1}$, we obtain $\Delta t_{\text {acc }} \sim 10^{3} \mathrm{~s}$, while for shorter binary separation, e.g. $a \sim 10^{10} \mathrm{~cm}(P \sim 5 \mathrm{~min}), \Delta t_{\mathrm{acc}} \sim 10^{2} \mathrm{~s}$, as validated by the results of our numerical integrations.

For instance, the NS in the system with $P=5$ min accretes $\approx 1 M_{\odot}$ in $\Delta t_{\text {acc }} \approx$ $100 \mathrm{~s}$. With the aid of Eq. (3), we estimate a difference in binding energies between a $2 M_{\odot}$ and a $3 M_{\odot}$ NS, i.e. $\Delta E_{\text {acc }} \approx 13 / 200\left(3^{2}-2^{2}\right) M_{\odot} c^{2} \approx 0.32 M_{\odot} c^{2}$ leading to a maximum luminosity $L_{\text {acc }} \approx 3 \times 10^{-} 3 M_{\odot} c^{2} \approx 0.1 \dot{M}_{b} c^{2}$. This accretion power, which could be as high as $L_{\text {acc }} \sim 0.1 \dot{M}_{b} c^{2} \sim 10^{47}-10^{51} \mathrm{erg} \mathrm{s}^{-1}$ for accretion rates in the range $\dot{M}_{b} \sim 10^{-6}-10^{-2} M_{\odot} \mathrm{s}^{-1}$, necessarily leads to signatures observable in long GRBs (see, e.g. Refs. 10 and 12).

\subsection{Post-explosion orbits and formation of NS-BH binaries}

We turn now to discuss the out-states of the IGC process. The SN explosion of the $\mathrm{CO}_{\text {core }}$ leaves as a central remnant, the $\nu \mathrm{NS}$, while the IGC process triggered by the hypercritical accretion of the SN ejecta onto the NS companion leads to the formation of a $\mathrm{BH}$. Thus, the question arises if BdHNe are natural sites for the formation of NS-BH binaries or if these binaries become disrupted during the $\mathrm{SN}$ explosion and the consequent IGC process. The answer to this question was recently given in Ref. 14, where it was shown that indeed most of BdHN form NS$\mathrm{BH}$ binaries since the high compactness of the orbit avoids the unbinding of the orbit.

In typical systems, most of the binaries become unbound during the SN explosion because of the ejected mass and momentum imparted (kick) on the newly formed compact object in the explosion of the massive star. Under the instantaneous explosion assumption, if half of the binary system's mass is lost in the SN explosion, the system is disrupted. In general, the fraction of massive binaries that can produce double compact object binaries is thought to be low: $\sim 0.001-1 \%{ }^{31-33}$

The mass ejected during the SN alters the binary orbit, causing it to become wider and more eccentric. Assuming that the mass is ejected instantaneously, the 
post-explosion semi-major axis is $a / a_{0}=\left(M_{0}-\Delta M\right) /\left(M_{0}-2 a_{0} \Delta M / r\right)$, where $a_{0}$ and $a$ are the initial and final semi-major axes respectively, $M_{0}$ is the total initial mass of the binary system, $\Delta M$ is the change of mass (equal to the amount of mass ejected in the $\mathrm{SN}$ ), and $r$ is the orbital separation at the time of explosion. ${ }^{34}$ For circular orbits, the system is unbound if it loses half of its mass. However, for very tight binaries as the one proposed in the IGC scenario, a number of additional effects can alter the fate of the binary.

The time it takes for the ejecta to flow past a companion in a SN is roughly $10-1000 \mathrm{~s}$. Although the shock front is moving above $10^{4} \mathrm{~km} \mathrm{~s}^{-1}$, the denser, lowervelocity ejecta can be moving at $10^{3} \mathrm{~km} \mathrm{~s}^{-1} .{ }^{12}$ The broad range of times arises because the SN ejecta velocities varies from $10^{2}-10^{4} \mathrm{~km} \mathrm{~s}^{-1}$. The accretion peaks as the slow-moving (inner) ejecta flows past the NS companion. For normal (wide) binaries, this time is a small fraction of the orbital period and the "instantaneous" mass-loss assumption is perfectly valid. However, in the compact binary systems considered in the IGC scenario, the orbital period ranges from only 100$1000 \mathrm{~s}$, and the mass loss from the SN explosion can no longer be assumed to be instantaneous.

We have seen how in BdHNe, the accretion process can lead to $\mathrm{BH}$ formation in a time-interval as short as the orbital period. We here deepen this analysis to study the effect of the SN explosion in such a scenario with a specific example of Ref. 14. Figure 4 shows as the ejecta timescale becomes just a fraction of the orbital timescale, the fate of the post-explosion binary is altered. For these models, we assumed very close binaries with an initial orbital separation of $7 \times 10^{9} \mathrm{~cm}$ in circular orbits. With $\mathrm{CO}_{\text {core }}$ radii of $1-4 \times 10^{9} \mathrm{~cm}$, such a separation is small, but achievable. We assume the binary consists of a $\mathrm{CO}_{\text {core }}$ and a $2.0 M_{\odot} \mathrm{NS}$ companion. When the $\mathrm{CO}_{\text {core }}$ collapses, it forms a $1.5 M_{\odot} \mathrm{NS}$, ejecting the rest of the core. We then vary the ejecta mass and time required for most of the ejected matter to move out of the binary. Note that even if $70 \%$ of the mass is lost from the system (the $8 M_{\odot}$ ejecta case), the system remains bound as long as the explosion time is just above the orbital time $\left(T_{\text {orbit }}=180 \mathrm{~s}\right)$ with semi-major axes of less than $10^{11} \mathrm{~cm}$.

The short orbits (on ejecta timescales) are not the only feature of these binaries that alters the post-explosion orbit. The NS companion accretes both matter and momentum from the SN ejecta, reducing the mass lost from the system with respect to typical binaries with larger orbital separations and much less accretion. In addition, as with common envelope scenarios, the bow shock produced by the accreting NS transfers orbital energy into the SN ejecta. Figure 4 shows the final orbital separation of our same three binaries, including the effects of mass accretion (we assume $0.5 M_{\odot}$ is accreted with the momentum of the SN material) and orbit coupling (30\% of the orbital velocity is lost per orbit). With these effects, not only do the systems remain bound even for explosion times greater than $1 / 2$ the orbital period but, if the explosion time is long, the final semi-major axis can be on par with the initial orbital separation. 

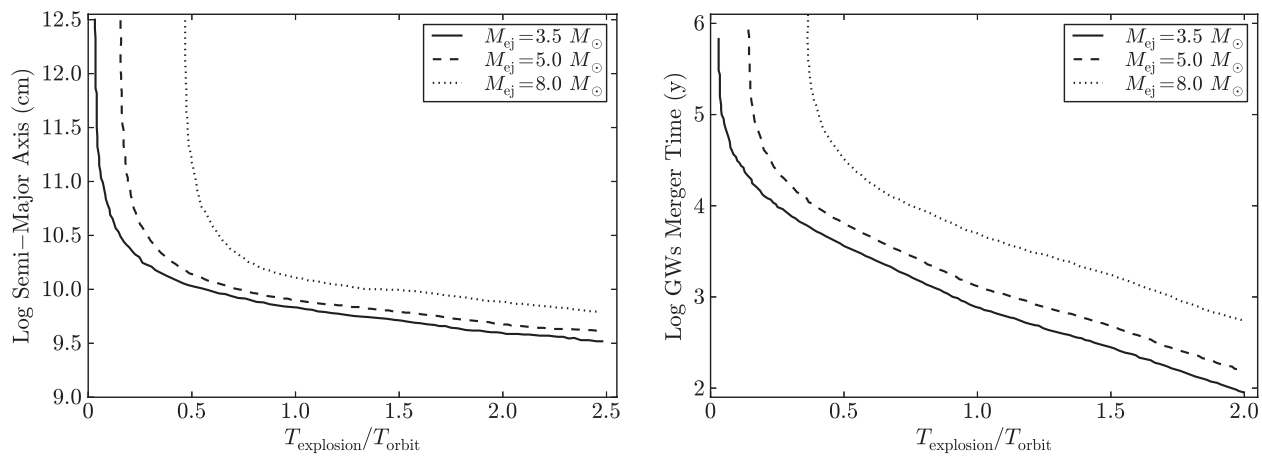

Fig. 4. Left panel: semi-major axis versus explosion time for three binary systems including mass accretion and momentum effects. Including these effects, all systems with explosion times above 0.7 times, the orbital time are bound and the final separations are on par with the initial separations. Right panel: merger time due to GW emission as a function of explosion time. Beyond a critical explosion time $\left(0.1-0.6 T_{\text {orbit }}\right.$ depending on the system), the merger time is less than roughly 10,000 yr. For most of our systems, the explosion time is above this limit and we expect most of these systems to merge quickly.

The tight compact binaries produced in these explosions will emit GW emission, ultimately causing the system to merge. For typical massive star binaries, the merger time is many Myr. For BdHNe, the merger time is typically 10,000 yr, or less, as shown in the right panel of Fig. 4. Since the merger should occur within the radius swept clean by the BdHN, we expect a small baryonic contamination around the merger site which might lead to a new family of events which we term ultrashort GRBs, U-GRBs. to this new family of events.

\section{NS-NS/NS-BH Mergers and Short GRBs}

Let us turn to short GRBs. We have mentioned that the most viable progenitors of short GRBs appear to be mergers of NS-NS and/or NS-BH binaries. Specifically, in the case of NS-NS mergers, the value of the critical mass of the NS, which crucially depends on the nuclear EOS, has been also found to be a most relevant parameter since it defines the fate of the post-merger object. ${ }^{8}$ In this section, we discuss the conditions that determine the fate of the NS-NS binary merger by estimating the mass and angular momentum of the post-merger object. Once we know these values, we can compare the mass of the merged core with the value of the NS critical mass obtained for uniformly rotating NSs. Based on this, we can asses whether a massive $\mathrm{NS}$ or a $\mathrm{BH}$ is formed from the merger.

We proceed to estimate the mass and the angular momentum of the post-merger core via baryonic mass and angular momentum conservation of the system. We adopt for simplicity that nonrotating binary components. We first compute the total baryonic mass of the NS-NS binary $M_{b}=M_{b_{1}}+M_{b_{2}}$ using the relation between the gravitational mass $M_{i}$ and the baryonic mass $M_{b_{i}}(i=1,2)$ recently obtained in Ref. 21 and given in Eq. (3) assuming $j_{\mathrm{NS}}=c J_{\mathrm{NS}} /\left(G M_{\odot}^{2}\right)=0$. The 
post-merger core will have approximately the entire baryonic mass of the initial binary, i.e. $M_{b \text {,core }} \approx M_{b}$, since little mass is expected to be ejected during the coalescence process. However, the gravitational mass of the post-merger core cannot be estimated using again the above formula since, even assuming nonrotating binary components, the post-merger core will necessarily acquire a fraction $\eta \leq 1$ of the binary angular momentum at the merger point. One expects a value of $\eta$ smaller than unity since, during the coalesce, angular momentum is lost, e.g. by gravitational wave emission and it can be also redistributed, e.g. into a surrounding disk.

To obtain the gravitational mass of the post-merger core, we can use again Eq. (3) relating the baryonic mass $M_{b, \mathrm{NS}}$ and the gravitational mass $M_{\mathrm{NS}}$ in this case with $j_{\mathrm{NS}} \neq 0$. The mass and angular momentum of the post-merger core, respectively $M_{\text {core }}$ and $J_{\text {core }}$, are therefore obtained from baryon mass and angular momentum conservation, i.e.

$$
M_{\text {core }}=M_{\mathrm{NS}}, \quad M_{b, \text { core }}=M_{b, \mathrm{NS}}=M_{b_{1}}+M_{b_{2}}, \quad J_{\text {core }}=J_{\mathrm{NS}}=\eta J_{\text {merger }},
$$

where $J_{\text {merger }}$ is the system angular momentum at the merger point. The value of $J_{\text {merger }}$ is approximately given by $J_{\text {merger }}=\mu r_{\text {merger }}^{2} \Omega_{\text {merger }}$, where $\mu=M_{1} M_{2} / M$ is the binary reduced mass, $M=M_{1}+M_{2}$ is the total binary mass, and $r_{\text {merger }}$ and $\Omega_{\text {merger }}$ are the binary separation and angular velocity at the merger point. If we adopt the merger point where the two stars enter into contact we have $r_{\text {merger }}=$ $R_{1}+R_{2}$, where $R_{i}$ is the radius (which depend on the EOS) of the $i$-component of the binary.

Given the parameters of the merging binary, the above equations lead to the merged core properties $M_{\text {core }}$ and $J_{\text {core }}$ ( or $j_{\text {core }}$ ). These values can be therefore confronted with the values of uniformly rotating, stable NSs to check if such a merger will lead either to a new massive NS or to an unstable merged core collapsing to a $\mathrm{BH}$.

For the sake of exemplifying, let us assume a mass-symmetric binary, $M_{1}=$ $M_{2}=M / 2$. In this case, Eq. (8) together with the above estimate of $J_{\text {merger }}$ lead to the angular momentum of the merged core $J_{\text {core }}=(\eta / 4)\left(G M^{2} / c\right) \mathcal{C}^{-1 / 2}$, where $\mathcal{C} \equiv$ $G M_{1} /\left(c^{2} R_{1}\right)=G M_{2} /\left(c^{2} R_{2}\right)$ is the compactness of the merging binary components. Therefore, if we adopt $M_{1}=1.4 M_{\odot}$ and $\mathcal{C}=0.15$ the above equations imply a merged core mass $M_{\text {core }}=(2.61,2.65) M_{\odot}$ for $\eta=(0,1)$, i.e. for a dimensionless angular momentum of the merged core $j_{\text {core }}=(0,5.06)$. Whether or not these pairs $\left(M_{\text {core }}, j_{\text {core }}\right)$ correspond to stable NSs depend on the nuclear EOS. A similar analysis can be done for any other pair of binary masses.

\section{Detectability of GWs Produced by the GRB Progenitors}

Having established the nature of the progenitors of each GRB sub-class, we turn now to briefly discuss the detectability of their associated GW emission. The minimum GW frequency detectable by the broadband aLIGO interferometer is 
$f_{\text {min }}^{\text {aLIGO }} \approx 10 \mathrm{~Hz} \cdot{ }^{35}$ Since during the binary inspiral, the GW frequency is twice the orbital one, this implies that a binary enters the aLIGO band for orbital periods $P_{\text {orb }} \lesssim 0.2 \mathrm{~s}$. Thus, $\mathrm{CO}_{\text {core-NS }}{ }^{-N i n a r i e s, ~ i n-s t a t e s ~ o f ~ X R F s ~ a n d ~ B d H N e, ~ a n d ~}$ $\mathrm{CO}_{\text {core }} \mathrm{BH}$ binaries, in-states of $\mathrm{BH}-\mathrm{SN}$, are not detectable by aLIGO since they have orbital periods $P_{\text {orb }} \gtrsim 5 \mathrm{~min} \gg 0.2 \mathrm{~s}$. Concerning their out-states after the corresponding hypercritical accretion processes, namely $\nu \mathrm{NS}-\mathrm{NS}$, out-states of XRFs, and $\nu \mathrm{NS}-\mathrm{BH}$, out-states of $\mathrm{BdHNe}$ and $\mathrm{BH}-\mathrm{SNe}$, they are not detectable by aLIGO at their birth but only when approaching the merger. Clearly, the analysis of the $\nu \mathrm{NS}-\mathrm{NS}$ mergers is included in the analysis of the S-GRFs and S-GRBs and, likewise, the merger of $\nu \mathrm{NS}-\mathrm{BH}$ binaries is included in the analysis of U-GRBs. In the case of NS-WD binaries, the WD is tidally disrupted by the NS making their GW emission hard to be detected (see, e.g. Ref. 36).

A coalescing binary evolves first through the inspiral regime to then pass over a merger regime, the latter composed by the plunge leading to the merger itself and by the ringdown (oscillations) of the newly formed object. During the inspiral regime, the system evolves through quasi-circular orbits and is well described by the traditional point-like quadrupole approximation. ${ }^{37-39}$ The GW frequency is twice the orbital frequency $\left(f_{s}=2 f_{\text {orb }}\right)$ and grows monotonically. The energy spectrum during the inspiral regime is: $d E / d f_{s}=(1 / 3)(\pi G)^{2 / 3} M_{c}^{5 / 3} f_{s}^{-1 / 3}$, where $M_{c}=\mu^{3 / 5} M^{2 / 5}=\nu^{3 / 5} M$ is the so-called chirp mass and $\nu \equiv \mu / M$ is the symmetric mass-ratio parameter. A symmetric binary $\left(m_{1}=m_{2}\right)$ corresponds to $\nu=1 / 4$ and the test-particle limit is $\nu \rightarrow 0$. The GW spectrum of the merger regime is characterized by a GW burst. ${ }^{40}$ Thus, one can estimate the contribution of this regime to the signal-to-noise ratio with the knowledge of the location of the GW burst in the frequency domain and of the energy content. The frequency range spanned by the GW burst is $\Delta f=f_{\mathrm{qnm}}-f_{\text {merger }}$, where $f_{\text {merger }}$ is the frequency at which the merger starts and $f_{\mathrm{qnm}}$ is the frequency of the ringing modes of the newly formed object after the merger, and the energy emitted is $\Delta E_{\text {merger }}$. With these quantities defined, one can estimate the typical value of the merger regime spectrum as: $d E / d f_{s} \approx \Delta E_{\text {merger }} / \Delta f$. Unfortunately, the frequencies and energy content of the merger regime of the above merging binaries are such that it is undetectable by LIGO. ${ }^{41}$

Since the GW signal is deep inside the detector noise, the signal-to-noise ratio $(\rho)$ is usually estimated using the matched filter technique. ${ }^{42}$ The exact position of the binary relative to the detector and the orientation of the binary rotation plane are usually unknown, thus it is a common practice to average over all the possible locations and orientations, i.e. ${ }^{42}:\left\langle\rho^{2}\right\rangle=4 \int_{0}^{\infty}\left\langle|\tilde{h}(f)|^{2}\right\rangle / S_{n}(f) d f=$ $4 \int_{0}^{\infty} h_{c}^{2}(f) /\left[f^{2} S_{n}(f)\right] d f$, where $f$ is the GW frequency in the detector frame, $\tilde{h}(f)$ is the Fourier transform of $h(t)$, and $\sqrt{S_{n}(f)}$ is the one-sided amplitude spectral density of the detector noise, and $h_{c}(f)$ is the characteristic strain, $h_{c}=$ $(1+z) /\left(\pi d_{l}\right) \sqrt{(1 / 10)\left(G / c^{3}\right)\left(d E / d f_{s}\right)}$. We recall that in the detector frame, the GW frequency is redshifted by a factor $1+z$ with respect to the one in the source frame, 
$f_{s}$, i.e. $f=f_{s} /(1+z)$ and $d_{l}$ is the luminosity distance to the source. We adopt a $\Lambda \mathrm{CDM}$ cosmology with $H_{0}=71 \mathrm{~km} \mathrm{~s}^{-1} \mathrm{Mpc}^{-1}, \Omega_{M}=0.27$ and $\Omega_{\Lambda}=0.73 .{ }^{43}$

A threshold $\rho_{0}=8$ in a single detector is adopted by LIGO. ${ }^{44}$ This minimum $\rho_{0}$ defines a maximum detection distance or GW horizon distance, say $d_{\mathrm{GW}}$, that corresponds to the most optimistic case when the binary is just above the detector and the binary plane is parallel to the detector plane. In order to give an estimate, the annual number of merging binaries associated with the above GRB subclasses detectable by aLIGO, we can use the lower and upper values of the aLIGO search volume defined by $\mathcal{V}_{s}=V_{\max }^{\mathrm{GW}} \mathcal{T}$, where $V_{\max }^{\mathrm{GW}}=(4 \pi / 3) \mathcal{R}^{3}$, where $\mathcal{T}$ is the observing time and $\mathcal{R}$ is the so-called detector range defined by $\mathcal{R}=\mathcal{F} d_{\mathrm{GW}}$, with $\mathcal{F}^{-1}=2.2627$ (see, Refs. 44 and 45 , for details). For a $(1.4+1.4) M_{\odot}$ NS binary and the three following different observational campaigns we have ${ }^{44}: 2015 / 2016$ $(\mathrm{O} 1 ; \mathcal{T}=3$ months $) \mathcal{V}_{S}=(0.5-4) \times 10^{5} \mathrm{Mpc}^{3} \mathrm{yr}, 2017 / 2018$ (O3; $\mathcal{T}=9$ months) $\mathcal{V}_{S}=(3-10) \times 10^{6} \mathrm{Mpc}^{3} \mathrm{yr}$, and the entire network including LIGO-India at design sensitivity $(2022+; \mathcal{T}=1 \mathrm{yr}) \mathcal{V}_{S}=2 \times 10^{7} \mathrm{Mpc}^{3} \mathrm{yr}$. The maximum possible sensitivity reachable in $2022+$ leads to $d_{\mathrm{GW}} \approx 0.2 \mathrm{Gpc}$, hence $V_{\max }^{\mathrm{GW}} \approx 0.033 \mathrm{Gpc}^{3}$, for such a binary. One can use this information for other binaries with different masses taking advantage of the fact that $d_{\mathrm{GW}}$ scales with the binary chirp mass as $M_{c}^{5 / 6}$. The expected GW detection rate by aLIGO can be thus estimated as: $\dot{N}_{\mathrm{GW}} \equiv \rho_{\mathrm{GRB}} V_{\text {max }}^{\mathrm{GRB}}$, where $\rho_{\mathrm{GRB}}$ is the inferred occurrence rate of GRBs shown in Table 1 computed in Ref. 6 . Bearing the above in mind, it is easy to check that there is a low probability for aLIGO to detect the GW signals associated with the GRB binary progenitors: indeed in the best case of the $2022+$ observing rung one obtains, respectively, $\sim 1$ detection every 3 and $5 \mathrm{yr}$ for U-GRBs and S-GRFs.

\section{Conclusions}

There is accumulated evidence on the binary nature of long and short GRBs. Such binaries are composed of $\mathrm{CO}_{\text {cores }}$, NSs, BHs and WDs in different combinations. We have here focused on the salient aspects of the NS physics relevant for the understanding of these binaries and their implications in GRB astrophysics, including their associated GW emission. We have discussed the crucial role of the NS critical mass in discriminating the GRB sub-classes. Therefore, we expect that the increasing amount of GRB high-quality data will help in constraining the NS critical mass with high accuracy with the most welcome result of constraining the NS matter content and the corresponding nuclear EOS.

\section{Acknowledgments}

M. Karlica, M. Kovacevic and Y. A. are supported by the Erasmus Mundus Joint Doctorate Program Grant Nos. 2013-1471, 2013-1471 and 2014-0707, respectively, from EACEA of the European Commission. M. M. and J. A. R. acknowledge the partial support of the Project No. 3101/GF4 IPC-11, and the target program F.0679 
of the Ministry of Education and Science of the Republic of Kazakhstan. C. C. and S. F. acknowledge INdAM-GNFM for support.

\section{References}

1. E. P. Mazets, S. V. Golenetskii, V. N. Ilinskii, V. N. Panov, R. L. Aptekar, I. A. Gurian, M. P. Proskura, I. A. Sokolov, Z. I. Sokolova and T. V. Kharitonova, Astrophys. Space Sci. 80 (1981) 3.

2. R. W. Klebesadel, The durations of gamma-ray bursts, in Gamma-Ray Bursts Observations, Analyses and Theories, eds. C. Ho, R. I. Epstein and E. E. Fenimore (Cambridge University Press, 1992).

3. J.-P. Dezalay, C. Barat, R. Talon, R. Syunyaev, O. Terekhov and A. Kuznetsov, AIP Conference Proceedings 265 (1991) 304-309.

4. C. Kouveliotou, C. A. Meegan, G. J. Fishman, N. P. Bhat, M. S. Briggs, T. M. Koshut, W. S. Paciesas and G. N. Pendleton, Astrophys. J. 413 (1993) L101.

5. M. Tavani, Astrophys. J. 497 (1998) L21.

6. R. Ruffini, J. A. Rueda, M. Muccino, Y. Aimuratov, L. M. Becerra, C. L. Bianco, M. Kovacevic, R. Moradi, F. G. Oliveira, G. B. Pisani and Y. Wang, Astrophys. J. 832 (2016) 136.

7. R. Ruffini, Y. Wang, M. Enderli, M. Muccino, M. Kovacevic, C. L. Bianco, A. V. Penacchioni, G. B. Pisani and J. A. Rueda, Astrophys. J. 798 (2015) 10.

8. R. Ruffini, M. Muccino, M. Kovacevic, F. G. Oliveira, J. A. Rueda, C. L. Bianco, M. Enderli, A. V. Penacchioni, G. B. Pisani, Y. Wang and E. Zaninoni, Astrophys. J. 808 (2015) 190.

9. R. Ruffini, M. G. Bernardini, C. L. Bianco, L. Vitagliano, S.-S. Xue, P. Chardonnet, F. Fraschetti and V. Gurzadyan, Black hole physics and astrophysics: The GRBSupernova connection and URCA-1-URCA-2, in The Tenth Marcel Grossmann Meeting, eds. M. Novello, S. Perez Bergliaffa and R. Ruffini (World Scientific, Singapore, 2006).

10. L. Izzo, J. A. Rueda and R. Ruffini, Astron. Astrophys. 548 (2012) L5.

11. J. A. Rueda and R. Ruffini, Astrophys. J. 758 (2012) L7.

12. C. L. Fryer, J. A. Rueda and R. Ruffini, Astrophys. J. 793 (2014) L36.

13. L. Becerra, F. Cipolletta, C. L. Fryer, J. A. Rueda and R. Ruffini, Astrophys. J. 812 (2015) 100.

14. C. L. Fryer, F. G. Oliveira, J. A. Rueda and R. Ruffini, Phys. Rev. Lett. 115 (2015) 231102 .

15. L. Becerra, C. L. Bianco, C. L. Fryer, J. A. Rueda and R. Ruffini, Astrophys. J. 833 (2016) 107.

16. J. Goodman, Astrophys. J. 308 (1986) L47.

17. B. Paczynski, Astrophys. J. 308 (1986) L43.

18. D. Eichler, M. Livio, T. Piran and D. N. Schramm, Nature 340 (1989) 126.

19. R. Narayan, T. Piran and A. Shemi, Astrophys. J. 379 (1991) L17.

20. E. Berger, Annu. Rev. Astron. Astrophys. 52 (2014) 43.

21. F. Cipolletta, C. Cherubini, S. Filippi, J. A. Rueda and R. Ruffini, Phys. Rev. D 92 (2015) 023007.

22. C. L. Fryer, W. Benz and M. Herant, Astrophys. J. 460 (1996) 801.

23. R. A. Chevalier, Astrophys. J. 346 (1989) 847.

24. Y. B. Zel'dovich, L. N. Ivanova and D. K. Nadezhin, Sov. Astron. 16 (1972) 209.

25. R. Ruffini and J. Wilson, Phys. Rev. Lett. 31 (1973) 1362. 
26. C. L. Fryer, F. Herwig, A. Hungerford and F. X. Timmes, Astrophys. J. 646 (2006) L131.

27. C. L. Fryer, Astrophys. J. 699 (2009) 409.

28. L. Izzo, R. Ruffini, A. V. Penacchioni, C. L. Bianco, L. Caito, S. K. Chakrabarti, J. A. Rueda, A. Nandi and B. Patricelli, Astron. Astrophys. 543 (2012) A10.

29. N. R. Tanvir, A. J. Levan, A. S. Fruchter, J. Hjorth, R. A. Hounsell, K. Wiersema and R. L. Tunnicliffe, Nature 500 (2013) 547.

30. N. R. Sibgatullin and R. A. Sunyaev, Astron. Lett. 26 (2000) 772.

31. C. L. Fryer, S. E. Woosley and D. H. Hartmann, Astrophys. J. 526 (1999) 152.

32. M. Dominik, K. Belczynski, C. Fryer, D. E. Holz, E. Berti, T. Bulik, I. Mandel and R. O'Shaughnessy, Astrophys. J. 759 (2012) 52.

33. K. A. Postnov and L. R. Yungelson, Living Rev. Rel. 17 (2014) 3.

34. J. G. Hills, Astrophys. J. 267 (1983) 322.

35. LIGO Scientific Collab. (J. Aasi et al.), Class. Quantum Grav. 32 (2015) 074001.

36. V. Paschalidis, M. MacLeod, T. W. Baumgarte and S. L. Shapiro, Phys. Rev. D 80 (2009) 024006.

37. P. C. Peters and J. Mathews, Phys. Rev. 131 (1963) 435.

38. P. C. Peters, Phys. Rev. 136 (1964) 1224.

39. M. Rees, R. Ruffini and J. A. Wheeler, Black Holes, Gravitational Waves and Cosmology (Gordon and Breach Science Publishers, New York, 1974).

40. M. Davis, R. Ruffini, W. H. Press and R. H. Price, Phys. Rev. Lett. 27 (1971) 1466.

41. R. Ruffini, J. Rodriguez, M. Muccino, J. A. Rueda, Y. Aimuratov, U. Barres de Almeida, L. Becerra, C. L. Bianco, C. Cherubini, S. Filippi, D. Gizzi, M. Kovacevic, R. Moradi, F. G. Oliveira, G. B. Pisani and Y. Wang, arXiv:1602.03545.

42. É. É. Flanagan and S. A. Hughes, Phys. Rev. D 57 (1998) 4535.

43. M. Rigault, G. Aldering, M. Kowalski, Y. Copin, P. Antilogus, C. Aragon, S. Bailey, C. Baltay, D. Baugh, S. Bongard, K. Boone, C. Buton, J. Chen, N. Chotard, H. K. Fakhouri, U. Feindt, P. Fagrelius, M. Fleury, D. Fouchez, E. Gangler, B. Hayden, A. G. Kim, P.-F. Leget, S. Lombardo, J. Nordin, R. Pain, E. Pecontal, R. Pereira, S. Perlmutter, D. Rabinowitz, K. Runge, D. Rubin, C. Saunders, G. Smadja, C. Sofiatti, N. Suzuki, C. Tao and B. A. Weaver, Astrophys. J. 802 (2015) 20.

44. B. P. Abbott et al., Living Rev. Rel. 19 (2016) 1.

45. L. S. Finn and D. F. Chernoff, Phys. Rev. D 47 (1993) 2198. 